\title{
MEDICAL MANAGEMENT OF PARKINSON'S DISEASE
}

C E Clarke

J Neurol Neurosurg Psychiatry 2002;72(Suppl I):i22-i27

$\mathrm{T}$ he management of Parkinson's disease has evolved rapidly over the last 10 years with the advent of new drugs, new classes of drug, and the resurgence of interest in surgery. Although there has been a move toward patients being cared for by neurologists or geriatricians with a special interest in the condition, along with a Parkinson's disease nurse specialist, for the foreseeable future most general neurologists will continue to treat patients with the condition.

The present review uses an evidence based approach to provide an update on the current place of medical treatment for Parkinson's disease. Unfortunately, in light of the lack of evidence in many areas, it is not always possible to be prescriptive and many treatment decisions must be left to the judgement of the individual clinician and the desires of patients.

\section{EARLY PARKINSON'S DISEASE}

The major issues regarding the difficulty in the clinical diagnosis of Parkinson's disease have been outlined already in this supplement (see p il0). It will be assumed in this review that the patient has idiopathic Parkinson's disease with significant functional disability that requires treatment.

\section{Neuroprotection}

The definitions of terms in relation to neuroprotection are outlined in table 1 and fig $1 .{ }^{1}$ The effects of this approach to treatment in Parkinson's disease can be measured in three ways:

- Comparison of the mortality rate on the putative neuroprotective agent with that in a control population within the setting of a randomised controlled trial (RCT)

- Measuring the decline in ${ }^{18} \mathrm{~F}$-fluorodopa uptake by dopaminergic neurones in the striatum using positron emission tomography (PET) to demonstrate reduced decline in the group treated with the neuroprotective agent

- During a total drug "wash out" period at the end of an RCT, rating scales (for example, unified Parkinson's disease rating scale (UPDRS), total or motor score) decline less in those treated with the neuroprotective agent.

All of these techniques suffer from significant problems. Demonstrating differences in mortality requires very large trials with national flagging to avoid missing the end point. PET is only available in a small number of centres so patients often need to travel distances to be assessed. The last method requires a complete wash out from all medication, including symptomatic treatments such as levodopa, that is rarely acceptable to patients. These and other issues have "dogged" the hunt for an effective neuroprotective agent in Parkinson's disease. Nevertheless, this must remain the "holy grail" of Parkinson's disease treatment as patients continue to die in excess of their peers. ${ }^{2}$

Selegiline was introduced as an adjuvant treatment in later disease, but a retrospective study raised the possibility that it might reduce mortality and thus be neuroprotective. This was in keeping with its known ability to inhibit the neurotoxicity of 1-methyl-4-phenyl-1,2,3,6tetrahydropyridine (MPTP) in experimental models of Parkinson's disease. This hypothesis was not confirmed by the DATATOP trial, and a UK Parkinson's Disease Research Group trial found increased mortality in those treated with selegiline, although a subsequent follow up analysis did not find a significant increase in mortality after correcting for baseline covariates. ${ }^{3}$ If selegiline does have a neuroprotective effect, this is likely to be small and will require much larger trials to prove.

Since the pathophysiological mechanism or mechanisms that cause Parkinson's disease are poorly understood, the rational development of neuroprotective agents has proved a difficult process. Neuroprotective strategies currently being accessed in clinical trials include the excitatory amino acid antagonist riluzole and the dopamine agonists pergolide and ropinirole. The excitatory amino acid glutamate, increased in the medial portion of the globus pallidus in Parkinson's disease, may be neurotoxic although no evidence of this is yet available in humans. Dopamine agonists reduce the accentuated compensatory turnover of dopamine which has been shown to be damaging in animal models. 

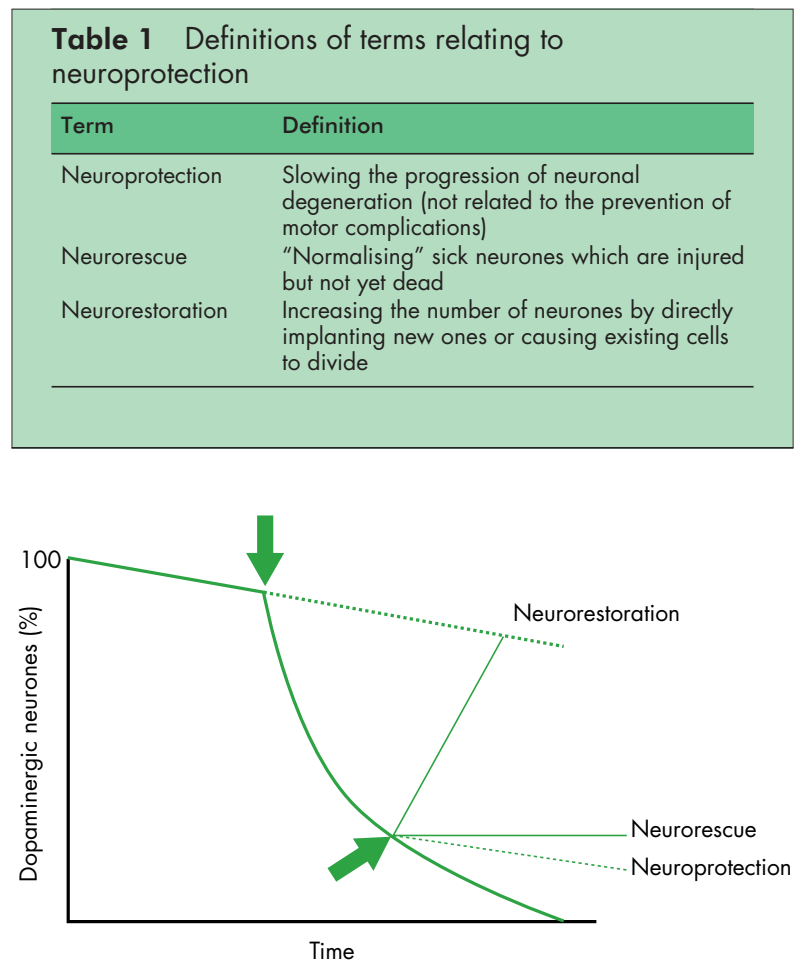

Figure 1 Schematic representation of neuroprotective strategies in Parkinson's disease. An insult at the time point indicated by the upper arrow leads to an acceleration in the normal age related loss of nigrostriatal dopaminergic neurones. Treatment is commenced at the point indicated by the lower arrow. Note: (1) in view of debate about the rates of decline in dopaminergic neurones no baseline for the $y$ axis has been given; (2) whether the decline in Parkinson's is exponential or linear, etc, is not known. Adapted from Parkinson's disease in practice, Royal Society of Medicine Press, 2001.

\section{Symptomatic treatment} Levodopa

The dramatic clinical effect of levodopa in Parkinson's disease was first demonstrated in the early 1970s and remains the "'gold standard" treatment for this condition. The impressive results and the urgent needs of patients meant that RCTs were not performed. The next significant development was the addition to levodopa of the peripheral dopa decarboxylase inhibitors, benserazide and carbidopa, that reduced peripheral side effects such as nausea, vomiting, and postural hypotension and also increased the delivery of levodopa to the brain. Consequently, levodopa preparations became widely prescribed in the UK in the 1980s. In recent years there has been a move away from levodopa as the first line treatment, at least for young patients, in view of its involvement in the generation of the long term motor complications and the possibility that it may be neurotoxic.

Motor complications comprise abnormal involuntary movements, including choreoathetoid dyskinesia, dystonia, and response fluctuations. The latter consist of a shortening response to each dose of medication (end-of-dose deterioration or wearing off effect) and unpredictable switching between the mobile "on" phase and relatively immobile "off" phase (on/off fluctuations). These affect $10 \%$ of patients with each year of levodopa treatment, so after five years of treatment $50 \%$ of patients will suffer from them. This rate of development of motor complications is worse in young onset patients (onset $<40$ years of age) with $100 \%$ suffering from these complications after six years of treatment.

\section{Abbreviations}

COMT: catechol-O-methyltransferase

DATATOP: deprenyl and tocopherol antioxidant therapy of parkinsonism

ELLDOPA: earlier versus later levodope

MAOB: monoamine oxidase $B$

MPTP: 1-methyl-4-phenyl-1,2,3,6-tetrahydropyridine

PET: positron emission tomography

RCT: randomised controlled trial

UPDRS: unified Parkinson's disease rating scale

Levodopa and dopamine are toxic to cultures of dopaminergic neurones. However, this is at supraphysiological doses and in the absence of glial cells that may inhibit neurotoxicity. There is no evidence that levodopa in vivo is neurotoxic, but this is currently the subject of a large ongoing North American trial (ELLDOPA trial).

The convincing relation between levodopa and motor complications has led to the move away from immediate release levodopa preparations as monotherapy. However, the alternatives considered below must live up to the efficacy and safety profile of levodopa.

\section{Modified release levodopa}

The possibility that the pulsatile stimulation produced by immediate release levodopa preparations may be responsible for motor complications led to the development of modified or slow release levodopa/decarboxylase inhibitor combinations (Madopar CR and Sinemet CR). However, two studies comparing these with immediate release levodopa failed to show any difference in motor complication rates after five years of such treatment. Since the modified release preparations are more expensive than traditional immediate release levodopa, there is little to recommend them at present in early disease.

\section{Anticholinergics}

Anticholinergics have been used in Parkinson's disease for more than 100 years, but it was the discovery of selective muscarinic antagonists with a more favourable side effect profile that led to their widespread use. Although many are available in the UK, benzhexol and orphenadrine are the most commonly used. A Cochrane systematic review of anticholinergic treatment in Parkinson's disease is underway. From the author's knowledge of the few trials in this area, anticholinergics are less effective than levodopa, apart from their beneficial effect on tremor, and have a worse adverse event profile, particularly in the elderly. Common central side effects are confusion, hallucinations, and transient cognitive impairment. Peripheral side effects include nausea, dry mouth, constipation, dizziness, blurred vision, precipitation of closed angle glaucoma, and urinary retention, especially in those with prostatic hypertrophy.

\section{Amantadine}

Initially investigated as an antiviral agent, amantadine was found by chance to be effective in Parkinson's disease. A Cochrane systematic review of the RCTs with amantadine is nearing completion but no comparative trials with levodopa have been performed. The clinical impression is that amantadine is less effective and the possibility that tolerance develops with long term treatment has been suggested by some. Like the anticholinergics, amantadine produces significant central side effects of confusion and hallucinations and peripheral 
Table 2 Summary of "modern" dopamine agonist monotherapy trials

\begin{tabular}{|c|c|c|c|c|c|}
\hline & & \multicolumn{4}{|l|}{ Agonist } \\
\hline & & Ropinirole & Pramipexole & Cabergoline & Pergolide \\
\hline \multirow{4}{*}{\multicolumn{2}{|c|}{$\begin{array}{l}\text { Date published } \\
\text { Patient numbers } \\
\text { Duration of trial (years) } \\
\text { Rescue levodopa allowed }\end{array}$}} & 1999 & 2000 & ? & ? \\
\hline & & 268 & 301 & 419 & 294 \\
\hline & & 5 & 2 & 5 & 3 \\
\hline & & Yes & Yes & Yes & No \\
\hline & Agonist & $20 \%$ * & $10 \% *$ & $22 \%$ * & $16 \% *$ \\
\hline & & & & v & v \\
\hline \multirow[t]{2}{*}{ Fluctuations incidence } & Agonist & NA & $24 \%$ & & \\
\hline & Levodopa & NA & $38 \%$ & $34 \%$ & $33 \%$ \\
\hline
\end{tabular}

reactions such as ankle oedema and livedo reticularis. Although the use of amantadine has declined in recent years, this may change if it is shown to be effective against dyskinesia in later disease. Several small trials have suggested that it may have such an effect, but much larger studies are required before it can be recommended for this use.

\section{Selegiline}

The possible neuroprotective properties of selegiline have been already considered. In terms of its symptomatic effects, in the DATATOP study selegiline delayed the need for levodopa by only nine months. Although no head-to-head comparison with levodopa has yet been performed, this suggests it has only a weak symptomatic effect compared with levodopa. It also has the disadvantage of causing significant sleep disturbance for some patients.

\section{Dopamine agonists}

The dopamine agonists were initially introduced as adjuvant therapy in late Parkinson's disease. However, the desire to delay the introduction of levodopa led to trials with agonists as monotherapy. The first trials of bromocriptine versus levodopa were reported in the mid 1980s and have recently been the subject of two Cochrane reviews. ${ }^{45}$ In summary, these showed that bromocriptine when used alone delayed the onset of dyskinesia but not motor fluctuations. In contrast, bromocriptine used in combination with levodopa (as levodopa sparing treatment) showed no clear advantage over levodopa. These results with bromocriptine were echoed in the two small trials with lisuride or combined lisuride/levodopa versus levodopa alone; lisuride must be used alone to delay the onset of motor complications.

Against this background, the manufacturers of the more recently introduced agonists each performed a single monotherapy trial with their agonist against levodopa (table 2). These showed significantly less motor complications in the agonist arms of each trial. However, in the published pramipexole ${ }^{6}$ and ropinirole ${ }^{7}$ studies those in the agonist arm had less improvement in motor impairments and/or disability (fig 2). This is surprising since these patients could have had the agonist dose titrated to the maximum tolerated and then open label levodopa could have been added. Presumably the patients were content for other reasons unrelated to motor impairments and disability. In terms of tolerability, hallucinations were significantly worse with ropinirole and somnolence with pramipexole. Withdrawals because of adverse events were greater with pergolide (18\%) than levodopa (10\%). Measures of health related quality of life may have given a more complete picture of patient's suffering in these studies.
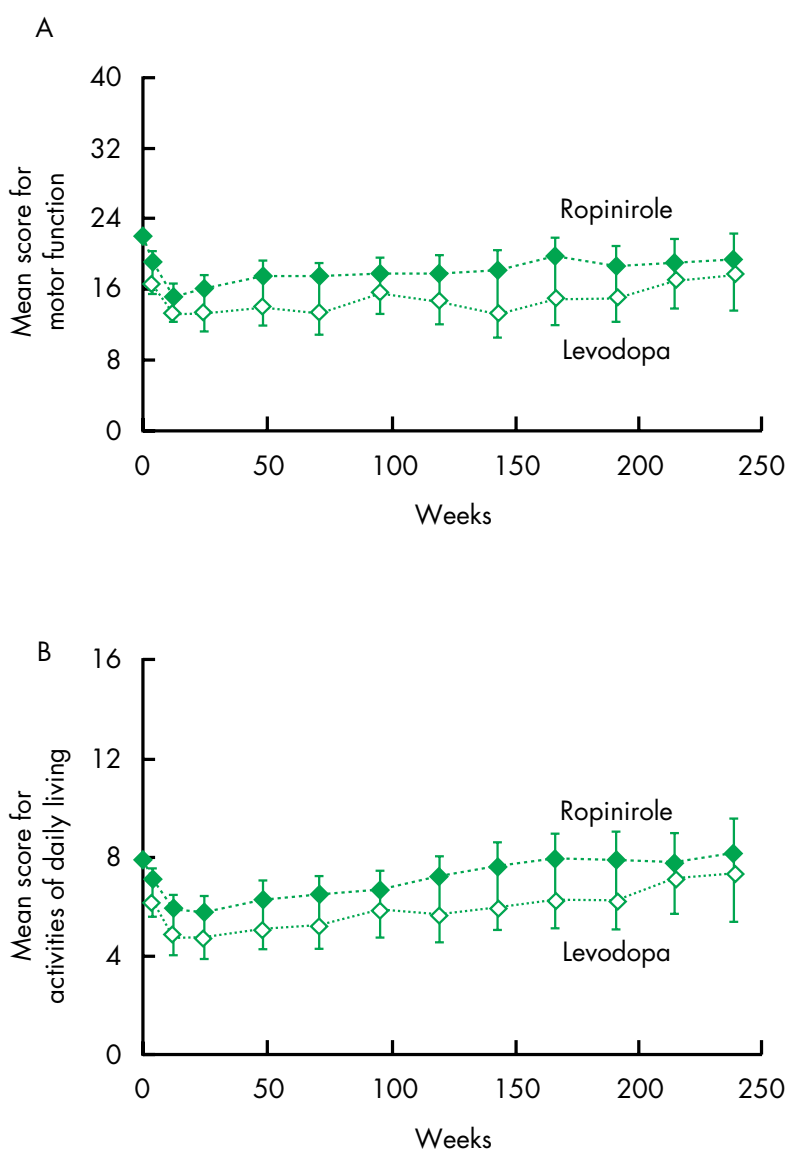

Figure 2 (A) UPDRS motor and (B) activities of daily living scores in the ropinirole versus levodopa study. Reproduced from Rascol and colleagues, ${ }^{7}$ with permission of the publisher.

In the pramipexole study up to the two year evaluation there was no difference in quality of life between the two arms.

It appears from the trial evidence that dopamine agonist monotherapy produces less motor complications than levodopa, but at the expense of more adverse events and possibly reduced efficacy. This appears to be a class effect although only one trial comparing the effects of the agonists has been performed. In this trial of ropinirole versus bromocriptine monotherapy with rescue levodopa, there was no difference in the incidence of motor complications between the two agonists although activities of daily living scores were significantly more improved in the ropinirole group. 


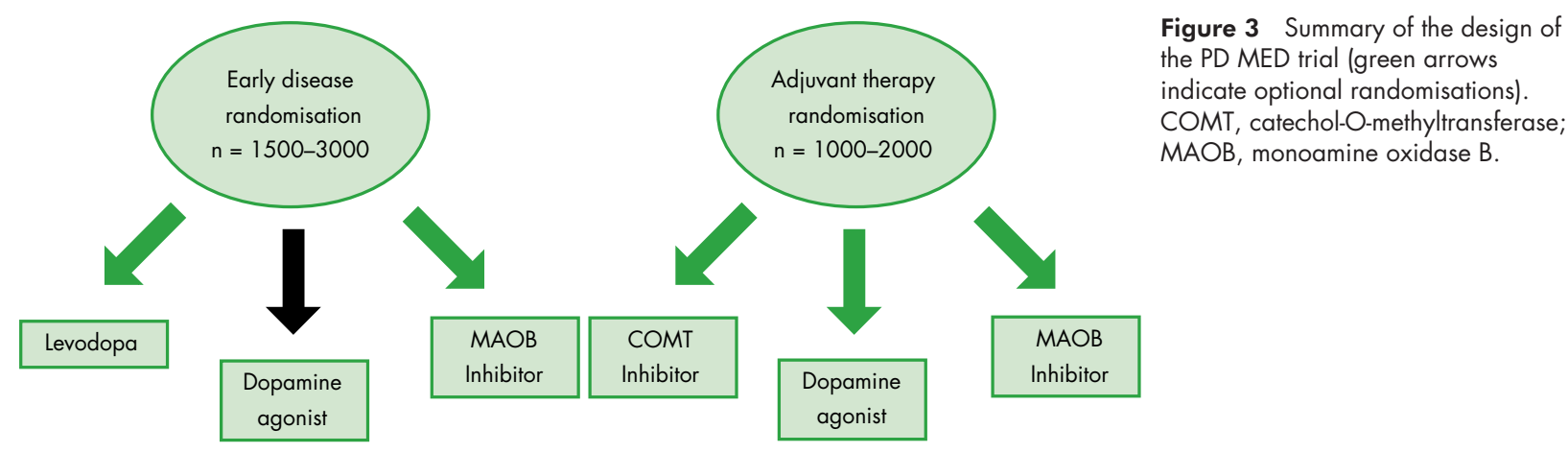

\section{Remaining issues in the pharmacotherapy of early Parkinson's disease}

In spite of the plethora of recent work addressing dopamine agonist monotherapy, many questions still remain. A change in practice to using agonist monotherapy for every new case of Parkinson's disease would double or treble the cost of treatment immediately. Is this worth it from the National Health Service's perspective in terms of cost effectiveness, and from the patient's perspective in terms of quality of life? Is agonist monotherapy neuroprotective and/or is levodopa toxic? It has been suggested that agonist monotherapy delays motor complications until levodopa is introduced, then complications accelerate until they are as severe as they would have been if an agonist had never been given. No data on this are available from the existing trials so this possibility remains. The recent agonist monotherapy trials included predominantly younger patients with Parkinson's disease: ropinirole-mean age 63 years; pramipexole-mean age 61 years; pergolide-mean age 59 years. So the results of these trials should not be generalised to the elderly population in whom further data are required before recommendations can be made. Similar questions are outstanding regarding selegiline monotherapy in terms of its effects on quality of life, cost effectiveness, and neuroprotection.

Many of these questions will be examined in the PD MED trial which is currently underway in the UK (fig 3). In the early disease 1500-3000 patients will be randomised to monotherapy with any levodopa preparation, any dopamine agonist, or any monoamine oxidase (MAO) B inhibitor (in practice selegiline at present). They will be followed for a minimum of five years with monitoring of quality of life, health economics, mortality, and the incidence of motor complications.

\section{LATER PARKINSON'S DISEASE}

In the "real" world general practitioners continue to diagnose and treat early Parkinson's disease with levodopa, only referring patients once motor complications have arisen. At this stage there is a choice of adjuvant treatment aimed at reducing patient's off time, reducing levodopa dose, and improving motor impairments and disability with acceptable side effects such as increased dyskinesia.

\section{Adjuvant treatment}

\section{Dopamine agonists}

The large number of RCTs examining agonist adjuvant treatment have been the subject of a number of Cochrane reviews by this author and others. The bromocriptine and lisuride versus placebo reviews failed to find sufficient evidence to reach any conclusions. The reviews of cabergoline, pergolide, pramipexole, and ropinirole versus placebo trials showed significant reductions in off time and levodopa dose with increased dyskinesia, but fewer withdrawals from the agonists. Other adverse events such as nausea, hallucinations, and somnolence were more frequent with the agonists. The recent concern about "sleep attacks" on pramipexole and ropinirole has raised awareness about the issue of somnolence, but this appears to be common with all dopaminergic treatment, even levodopa. No clear differences between the agonists were apparent from this meta-analysis, although this conclusion should be treated with caution since the agonists were not directly compared.

From the perspective of clinical practice active comparitor trials are more valuable. Most of the "modern" agonists have been compared with bromocriptine in RCTs. Cochrane reviews of these have shown that cabergoline, pramipexole, and ropinirole produce 30 minutes more reduction in off time than bromocriptine, but otherwise there is no difference in the efficacy or safety of modern agonists compared with bromocriptine. Whether this additional reduction in off time with modern agonists is worth the additional cost cannot be calculated using health economics models as insufficient data are available in the UK on the "cost" of off time. This work has also allowed sample size calculations to be performed which show that trials of multiple agonists as adjuvant treatment would be prohibitively large (around 5000 patients) and expensive. Therefore decisions regarding which agonist to use cannot be based on efficacy or safety, but rather on issues such as dose frequency, ease of titration, and affordability.

In later Parkinson's disease, when off periods become frequent and unpredictable in spite of optimal oral treatment, apomorphine injections or infusion can be used. ${ }^{8}$ Apomorphine is a potent dopamine agonist which is extensively metabolised in the liver, thus having poor oral bioavailability. Most of the data on apomorphine have come from small nonrandomised and uncontrolled studies, although a recent small placebo controlled study has confirmed the benefits of intermittent apomorphine injections. Apomorphine infusions cost around $£ 10000$ per patient per year, although this may be offset by reductions in other costs such as residential care. Both intermittent injections and continuous infusions should be confined to specialist movement disorders clinics where clinicians and Parkinson's disease nurse specialists have experience with the techniques involved. 
Selegiline

No systematic review of selegiline trials is available, but from the author's knowledge trials were small and of short duration with no measures of off time. Nevertheless they support selegiline reducing levodopa dose and improving motor impairments. While many clinicians believe selegiline is less effective than the dopamine agonists no evidence exists to support this view.

In recent years a new formulation of selegiline has become available. The oral fast-melt Zydis preparation avoids first pass metabolism in the liver, and thus produces fewer amphetamine-like metabolites and possibly fewer side effects. The single randomised placebo controlled trial available showed similar reductions in off time as with agonists on the $2.5 \mathrm{mg}$ /day dose and significant reductions in motor impairments and disability.

\section{COMT inhibitors}

Catechol-O-methyltransferase (COMT) inhibitors reduce the metabolism of levodopa to 3-O-methyldopa in the periphery thereby increasing the amount crossing the blood-brain barrier. This produces a $30-50 \%$ increase in levodopa half life and a $25-100 \%$ increase in the levodopa concentration versus time curve, while leaving the maximum plasma concentration of levodopa unchanged. Tolcapone was the first COMT inhibitor licensed in the UK, but was withdrawn on account of several cases of fatal hepatic toxicity. Entacapone does not seem to suffer from this problem. Although clinical experience suggests that entacapone is not as effective as both agonists and tolcapone, the evidence from placebo controlled trials shows similar improvements in on time, off time, and levodopa dose as with dopamine agonists. Insufficient data are available on activities of daily living and motor impairments to reach any firm conclusion. Entacapone produced dopaminergic adverse events that were reduced by levodopa dose reduction, along with unexplained diarrhoea and discolouration of urine. A Cochrane review of COMT inhibitors is awaited.

\section{Modified release levodopa}

RCTs in patients with motor complications showed that Sinemet CR and Madopar CR produced moderate reduction in off time compared with immediate release preparations, but variable effects on dyskinesia with a tendency for these to increase. The doses of both modified release preparations had to be increased by $20-60 \%$ because of reduced absorption and bioavailability. Adverse events were similar for immediate and modified release agents. Modified release levodopa also allowed a reduction in the number of daily doses compared with the standard formulation from an average of five to between three and four doses per day. In these trials, clinicians and patients preferred modified release to the immediate release preparation, although the results in general clinical practice have been less convincing.

\section{Remaining issues in the pharmacotherapy of later Parkinson's disease}

Although many believe dopamine agonists to be the most effective agents to use as adjuvant therapy, this conclusion is not evidence based. We require further data comparing agonists, COMT inhibitors, and selegiline in terms of quality of life and health economics. This is the subject of the second part of the PD MED trial (fig 3). Further information is also required on when to use apomorphine infusions, particularly in relation to surgical treatment. Answers should be generated in part from the PD SURG trial (see p 00).

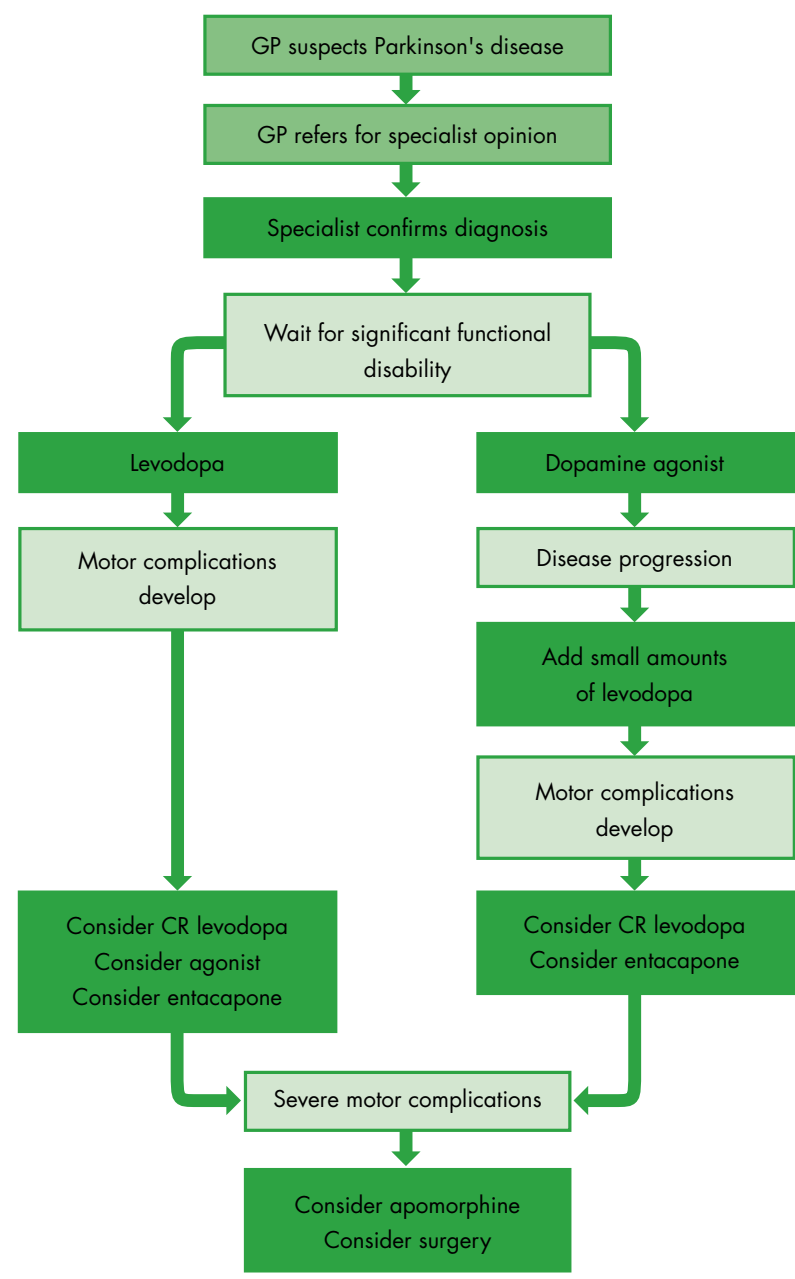

Figure 4 Treatment algorithm for Parkinson's disease. CR, controlled release; GP, general practitioner. Adapted from Bhatia and colleagues, ${ }^{9}$ with permission of the publisher.

\section{PARAMEDICAL THERAPIES}

Paramedical therapies have traditionally been used in later Parkinson's disease when significant functional disability has developed. Long waiting times for treatment for neurological patients, combined with a perceived lack of evidence of efficacy, has led to low referral rates in the UK despite the enthusiastic support of patients. RCTs of the main three paramedical therapies have recently been the subject of a series of Cochrane systematic reviews by the author and colleagues. There was insufficient evidence of the efficacy of physiotherapy, occupational therapy, or speech and language therapy for dysarthria or dysphagia in Parkinson's disease. It must be emphasised that lack of evidence does not mean lack of efficacy. Further trials are required to decide whether and when these treatments should be used. The UK Parkinson's Disease Society is in the process of developing large pragmatic trials of both physiotherapy and occupational therapy.

\section{PARKINSON'S DISEASE NURSE SPECIALISTS}

The development of specialist peripatetic nurses for Parkinson's disease has been led by the Parkinson's Disease Society in the UK. From small beginnings, around 100 are now funded from various sources including the NHS. The nurses have a number of roles but they primarily act as a key worker for the patient with Parkinson's disease, liaising with therapists, social services, the primary and secondary care teams, etc. 
They serve to educate the patient, relatives, and members of the medical and allied professions about the condition. Their ability to help the patient with complex changes in medication, including apomorphine infusions, within set limits can be invaluable. Parkinson's disease nurse specialists have been the subject of a recent large RCT which showed significant improvements in an open label patient global impression scale at no additional cost to the NHS. No advantages in quality of life or mortality were found, but the study was confounded by selegiline withdrawal.

\section{SPECIALIST CLINICS}

Although over the last 10 years there has been a move towards specialist movement disorders or Parkinson's disease clinics, there is no evidence that they provide better care than standard neurology or geriatrics clinics. However, a recent study from Wales showed that the diagnostic error rate in parkinsonian syndromes was over 50\% in general practice. Brain bank studies show an error rate of around 25\% in neurologist's hands, but this reduced to only $8 \%$ in the DATATOP study in which committed clinicians with a particular interest in Parkinson's disease took part. This circumstantial evidence suggests that, at least from the diagnostic point of view, specialist clinics may be worthwhile.

\section{GUIDELINES}

Several sets of guidelines for the management of Parkinson's disease have been produced in the $\mathrm{UK}^{9}$ and the USA, ${ }^{10}$ but these failed to use a rigorous procedure to sift the evidence before a panel of experts synthesised such data and made their recommendations. For the moment these should be treated with some caution. The treatment algorithm from a UK guidelines panel is shown in fig 4.

Conflict of interest: Dr Clarke has received payment from the manufacturers of several of the agents discussed in this review for lecture and consultancy fees.

\section{REFERENCES}

1 Schapira AHV. Parkinson's disease. BM 1999;318:311-14.

Contains a valuable summary of issues regarding neuroprotection. 2 Clarke CE. Does levodopa therapy delay death in Parkinson's disease? A review of the evidence. Mov Disord 1995;10:250-6.

3 Ben-Shlomo Y, Churchyard A, Head J, et al. Investigation by Parkinson's Disease Research Group of United Kingdom into excess mortality seen with combined levodopa and selegiline treatment in patients with early, mild Parkinson's disease: further results of randomised trial and confidential inquiry. BM 1998;316:1191-6.

4 Ramaker C, van Hilten J. Bromocriptine versus levodopa in early Parkinson's disease. The Cochrane Library. Oxford: Update Software, 2000.

- One of the many useful systematic reviews of treatment in the Cochrane Library.

5 Ramaker C, van Hilten J. Bromocriptine/levodopa versus levodopa in early Parkinson's disease. Parkinsonism and Related Disorders 1999;5(suppl):82.

6 Parkinson Study Group. Pramipexole versus levodopa as initial treatment for Parkinson's disease. JAMA 2000;284:1931-8.

7 Rascol O, Brooks D, Korczyn A, et al. A five-year study of the incidence of dyskinesia in patients with early Parkinson's disease who were treated with ropinirole or levodopa. N Engl J Med 2000;342: 1484-91.

Two pivotal monotherapy trials with modern dopamine agonists.

8 Frankel JP, Lees AJ, Kempster PA, et al. Subcutaneous apomorphine in the treatment of Parkinson's disease. J Neurol Neurosurg Psychiatry 1990;53:96-101.

9 Bhatia K, Brooks D, Burn D, et al. Guidelines for the management of Parkinson's disease. Hospital Medicine 1998;59:469-80

10 Olanow C, Watts R, Koller W. An algorithm (decision tree) for the management of Parkinson's disease (2001): treatment guidelines. Neurology 2001;56(suppl 5):S1-88

Two sets of management guidelines presenting therapeutic options but few answers.

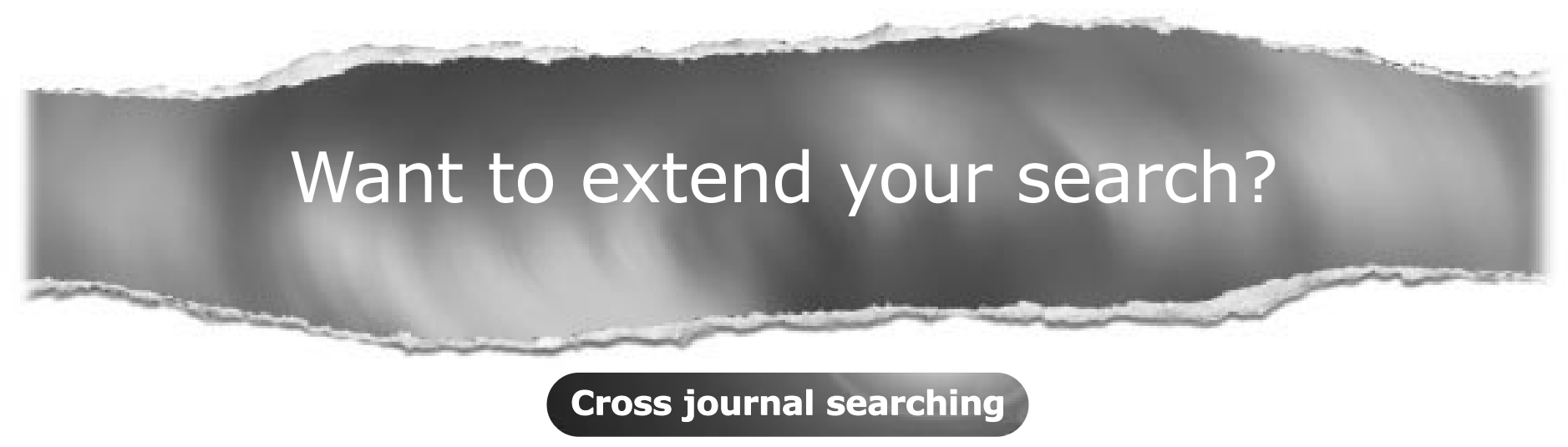

If you can't find what you are looking for in the Journal of Neurology, Neurosurgery, and Psychiatry you can extend your search across many of the more than 200 journals available for selection. You can restrict your search to specific subject areas (eg, clinical medicine, basic research), or select specific journals, or search all available titles.

\section{www.jnnp.com}

